\title{
A Case Report of Thoracic Outlet Syndrome with Vascular Complications due to Cervical Rib
}

\author{
MF Hossain, AH Parvez, MN Sabah, AHM Bashar, NC Mandal, E Hakim, S Samad \\ Department of Vascular Surgery, NICVD, Dhaka
}

\begin{abstract}
:
Keywords:

Thoracic outlet

syndrome,

Cervical rib, thromboembolism.

Thoracic outlet syndrome (TOS) is recognized in approximately $8 \%$ of the population. Its manifestations may be neurologic or vascular or both, depending on the component of the neurovascular bundle predominantly compressed. The diagnosis is suspected from the clinical picture and investigations. Treatment is initially conservative but persistence of significant symptoms, which occurs in approximately $5 \%$ of patients with diagnosed TOS, is an indication for cervical rib and or first rib resection. ${ }^{1}$ We report the case of a young woman having a left sided cervical rib. She had presented with clinical features of both neurogenic and arterial TOS. Surgical resection of the cervical rib was performed uneventfully through a supraclavicular approach which resulted in complete relief of her symptoms.
\end{abstract}

(Cardiovasc. j. 2014; 7(1): 63-65)

\section{Introduction:}

Cervical rib is development of the 7 th cervical vertebra more than $2.5 \mathrm{~cm}$ which is normal and symptomless. It may take the form of a fibrous band stretching down to the first rib. It lies either behind or below the brachial plexus and Subclavian vessels and exerts pressure on them during movements of the upper limb. Symptoms are usually delayed until adolescence because pressure on the brachial plexus by the rib is not felt until a lowering of the shoulder girdle, which occurs about that age, takes place. It is seen especially in females who have an adolescent increase in weight of the shoulders and breasts. Symptoms are commoner in people with long necks and sloping shoulders than in those with square built. The rib is not commonly palpable.

In 1740, Hunauld reported the first case of cervical rib. In1869, Gruber developed the methods for diagnosis. The first unsuccessful resection of cervical rib in a patient with thoracic outlet syndrome (TOS) was performed by Coote in 1861. In 1905, Murphy first made a successful resection of cervical rib in the patient with TOS and he also removed the first rib using supraclavicular approach for the first time. In 1927, Adson Coffey emphasized the role of ligaments, soft tissues and scalenus muscle in producing TOS and made the first successful resection of the scalenus anticus muscle. ${ }^{1,2}$

\section{Case Report:}

A 15-year-old female presented with a swelling over the left side of the neck with occasional pain along the left side of the neck, left arm and forearm for 6 months especially on raising the arm. She gave no history of trauma to the neck or arm. She also didn't experience coldness, fatigue or bluish discoloration of left upper limb.

On examination, a visible swelling (Fig.1) was present on left posterior triangle of the neck

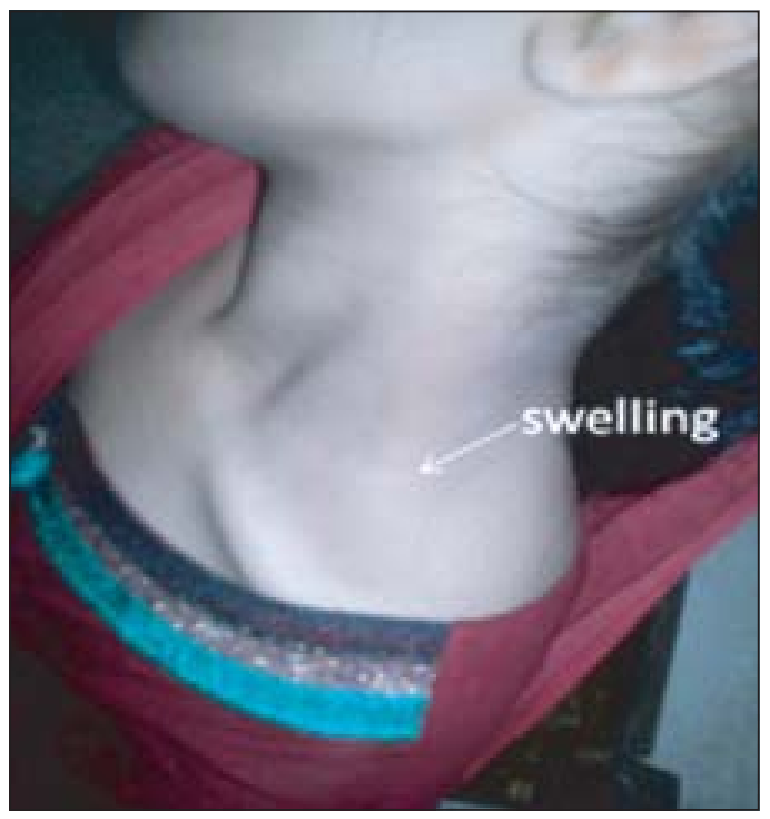

Fig.-1: Photograph showing a left supraclavicular swelling.

Address of Correspondence: Dr. Md. Fidah Hossain, Department of Vascular Surgery, National Institute of Cardiovascular Diseases, Dhaka, Bangladesh. E-mail: fidah.hossain@gmail.com 
with palpable bony prominence on left lateral neck. Local tenderness was absent but the patient complained of pain on raising the left upper limb. Palpable thrill was present over left supraclavicular region. Peripheral pulses were normal, muscle wasting was absent, motor and sensory functions were intact. On auscultation, bruit could be heard over the left supraclavicular region. Adson's scalene test, costoclavicular test and hyperabduction test were negative.

X-ray cervical spine showed bilateral cervical rib with the left sided rib extending up to the first rib forming an attachment with the latter. (Fig.2)

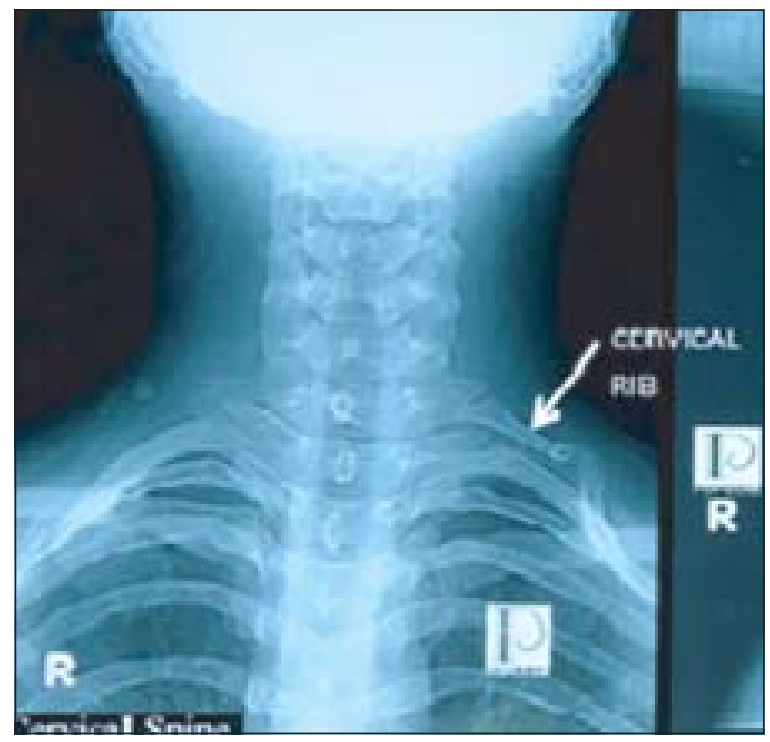

Fig.-2: Plain X-ray showing the left sided cervical rib.

CT Angiogram of the aortic arch and the left upper extremity revealed a common origin of the Brachiocephalic trunk and left common carotid artery. Left subclavian artery was compressed for a length of about $5 \mathrm{~mm}$ between the cervical rib and the first rib. The artery had a post-stenotic dilatation $(8.8 \mathrm{~mm})$ and formed pseudarthrosis with the cervical rib (Fig.3). She was diagnosed as a case of TOS due to bilateral cervical rib with post stenotic dilatation of left subclavian artery.

On 23.09.13, she was operated under local anesthesia with Interscalene Brachial Plexus Block. The approach was supraclavicular. At operation, the cervical rib was seen sticking up

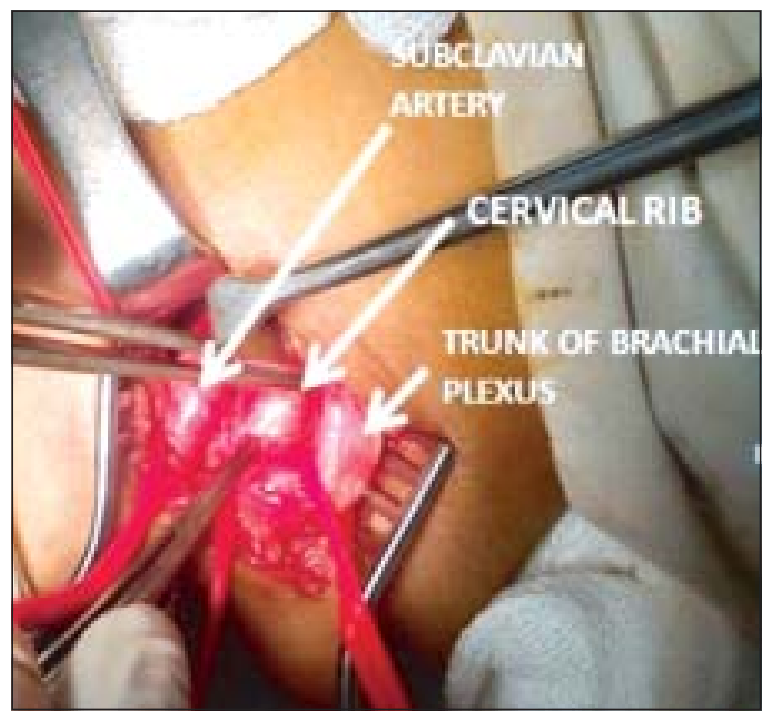

Fig.-3: Intraoperative photograph showing major structures in the field.

like an inverted wish-bone, articulating with the 7th cervical vertebra behind and also with an upward projection of bone from the first rib in front. Brachial plexus and Subclavian artery were found compressed between scalene muscle and cervical rib. Release of anterior scalene muscle was done. Post-stenotic dilatation of Subclavian artery was present but there were no aneurysm or thrombosis. So no intervention was done to Subclavian artery. Removal of the cervical rib was then undertaken in a piece-meal manner excluding the periosteum.

Postoperative recovery was good. Symptoms improved immediately after the operation. On the first post operative day, there was mild pain and numbness in the entire left arm. Intermittent shoulder exercise was started on the second post operative day. The patient was almost completely free from symptoms 4 weeks after the operation.

\section{Discussion:}

TOS/Cervical Rib Syndrome: TOS- a term coined by Rob and Standover, refers to compression of the subclavian vessels and brachial plexus at the superior aperture of the chest. It was previously designated, according to presumed etiologies, as Scalenus anticus, Costoclavicular, Hyperabduction, Cervical rib and First thoracic rib syndromes. The various syndromes are similar and the compression mechanism is often difficult 
to identify. ${ }^{1} 70 \%$ cervical ribs occur in females. ${ }^{3}$ Neurogenic variety constitutes $95 \%$ of all cases of TOS, Venous TOS comprises 2-3\% and Arterial TOS $<1 \%{ }^{3}$

Symptoms of neurogenic TOS includes pain in the neck and shoulder, paresthesia and numbness over median and ulnar nerve dermatome resulting from traction and compression of $\mathrm{C} 8$, T1 nerve root (lower trunk). Diagnosis and treatment of neurogenic TOS is most difficult.

Clinical features of arterial TOS include pain, claudication, pallor, Raynaud's phenomenon, peripheral embolism etc. and those of venous TOS include coolness, duskiness, venous dilation, edema, subclavian vein thromboembolism and peripheral necrosis. ${ }^{1,4}$

The presenting symptom in the present case was pain along the left side of the neck and left upper limb. A left supraclavicular swelling was found on clinical examination which turned out to be a post-stenotic dilatation of the SCA. There was no sign of arterial insufficiency or peripheral thrombo-embolism and the patient was neurologically intact. Unfortunately, a vast majority of patients suffering from arterial TOS in this country present with advanced features of arterial insufficiency most often in the form of gangrenous fingers or sometimes with gangrene of the entire hand or forearm. In these patients, some form of amputation becomes necessary along with the surgical treatment of arterial TOS.

Our decision to leave alone the dilated segment of the SCA in this case may be a contentious one. It has been found that such dilated segments of the artery returns to normal dimension over time once the proximal stenotic element has been removed. ${ }^{5,6}$ However, we do perform surgical repair of the aneurysmal SCA whenever indicated. Aneurysmorrhaphy is our most common strategy although venous or prosthetic interposition grafting is also performed in appropriate cases. Embolectomy for distal thrombo-embolism is another important step in this surgery. The result of embolectomy, however, is not uniformly rewarding. Recurrent thrombosis or embolism is particularly common in cases presenting late.

Some form of neuropraxia is almost a rule in the immediate post-operative period because of manipulation of the brachial plexus. Extremely variable in severity, the complication can be minimized by gentle handling of the trunks of the brachial plexus. Physiotherapy and medications are usually sufficient for alleviation of the symptoms of neuropraxia.

Though extremely rare, regeneration of cervical rib does occur specially when denudation of the periosteal layer remains incomplete. Thus particular care should be taken to strip the periosteum before excision of the rib.

In conclusion, we reported here the case of a young woman with arterial TOS who presented early without signs of arterial insufficiency and was benefitted from the surgical treatment.

\section{Conflict of Interest - None.}

\section{References:}

1. Harold C. Urschel, Jr. and Amit N. Thoracic Outlet Syndrome and Dorsal Sympathectomy. In: F.W. Sellke, P.J.D. Nido, and S.J. Swanson. Eds. Sabiston \& Spencer Surgery of the Chest, 8th edition. Philadelphia: Elsevier Saunders, 2010: 389-408.

2. Murchite, Sheetal. Gaikwad, V.V. Chaudhuri, Saikat. Kulkarni, R.M. Cervical Rib: A Case Report with Review of Literature. Indian Journal of Applied Research 2013;10:1-3.

3. Sanders RJ. Thoracic Outlet Syndrome: General Considerations. In: Cronewell JL, Johnston KW. Eds. Rutherford's Vascular Surgery. Philadelphia: Saunders Elsvier; 2010: 1865-1917.

4. Gerdes J. Thoracic Outlet Syndrome. Available at www.medschool.lsuhsc.edu/surgery/docs/grand rounds tos.ppt [Accessed 01/10/2013].

5. Kim, S.S. Ryu, H.C. (2005). Cervical Rib Syndrome- A Case Report. Available at www.kpos.or.kr/event/200501/ 200501kss.ppt [Accessed 01/10/2013].

6. Urschel HC Jr, Razzuk MA . Neurovascular compression in the thoracic outlet: changing management over 50 years. Ann Surg 1998;228(4):609-17. 